PROCEEDINGS OF THE

AMERICAN MATHEMATICAL SOCIETY

Volume 134, Number 7, Pages 1995-2002

S 0002-9939(05)08236-5

Article electronically published on December 19, 2005

\title{
THE INNER AMENABILITY OF THE GENERALIZED THOMPSON GROUP
}

\author{
GABRIEL PICIOROAGA
}

(Communicated by David R. Larson)

\begin{abstract}
In this paper we prove that the general version $F(N)$ of the Thompson group is inner amenable. As a consequence we generalize a result of P. Jolissaint. To do so, we prove first that $F(N)$ together with a normal subgroup are i.c.c (infinite conjugacy classes) groups. Then, we investigate the relative McDuff property out of which we extract property $\Gamma$ for the group von Neumann algebras involved. By a result of E. G. Effros, $F(N)$ follows inner amenable.
\end{abstract}

\section{INTRODUCTION}

The Thompson group $F$ can be regarded as the group of piecewise-linear, orientation-preserving homeomorphisms of the unit interval which have breakpoints only at dyadic points and on intervals of differentiability the slopes are powers of two. The group was discovered in the 1960s by Richard Thompson and in connection with the now celebrated groups $T$ and $V$ it led to the first example of a finitely presented infinite simple group. Since then, these groups have received considerable applications in such fields as homotopy theory or operator algebras. The group $V$ has been generalized by Higman $([\mathrm{Hig}])$ and $F$ by Brown $([\mathrm{Br}])$ and M. Stein $([\underline{\mathrm{St}}])$. The generalized Thompson group we study in this paper corresponds to $F(p)$ in St (or $F_{p, \infty}$ in $\mathrm{Br}$ ).

In 1979 Geoghegan conjectured that $F$ is not amenable. This problem is still open and of great importance for group theory.

Inner amenability (a larger property than amenability even though the definition is just a slight variation of the amenability one) was introduced by Effros. He also observed that if the group von Neumann algebra corresponding to a group $G$ is a $I I_{1}$ factor having the property $\Gamma$ of Murray and von Neumann, then $G$ is inner amenable. In [Jol1] Jolissaint proved that the Thompson group is inner amenable, then in [Jol] he proved more: the $I I_{1}$ factor associated with the Thompson group has the so-called relative McDuff property; in particular, property $\Gamma$ is satisfied.

In this paper we prove that for any integer $N \geq 2$ the generalized Thompson group $F(N)$ satisfies the relative McDuff property and therefore it is inner amenable, hence the title. To prove the McDuff property we will use two results from [Jol]. We remark that inner amenability could be proved directly using the ideas in [Jol1] and the infinite presentation of $F(N)$; however, the relative McDuff

Received by the editors February 8, 2005.

2000 Mathematics Subject Classification. Primary 46K10, 22 D15.

(C)2005 American Mathematical Society 
property is a stronger result. In the next section we prepare some basics on the Thompson groups and group von Neumann algebras. In the last section we prove the main result of the paper and its corollary. We conclude with a question whose answer might connect the theory of $I I_{1}$ factors and the (non)amenability of $F$.

\section{BACKGROUND}

Definition 2.1. For $N \in \mathbb{N}, N \geq 2$, the Thompson group $F(N)$ is the set of piecewise linear homeomorphisms from the closed unit interval $[0,1]$ to itself that are differentiable except at finitely many $N$-adic rationals and such that on intervals of differentiability the derivatives are powers of $N$.

In $[\mathrm{Br}]$ finite and infinite presentations of $F(N)$ are given. For example,

$$
F(N)=\left\langle x_{0}, x_{1}, \ldots, x_{i}, \ldots \mid x_{j} x_{i}=x_{i} x_{j+N-1}, i<j\right\rangle .
$$

We will not make use of them here, our arguments being based on some special elements of $F(N)$ (see DutP]

$$
A_{d, p}(x)=\left\{\begin{array}{lr}
x / N^{p}, & 0 \leq x \leq d, \\
x-d+d / N^{p}, & d \leq x \leq 1-d / N^{p}, \\
N^{p} x+1-N^{p}, & 1-d / N^{p} \leq x \leq 1,
\end{array}\right.
$$

where $d$ is an $N$-adic, $p \in \mathbb{Z}$ such that $d / N^{p}<1$.

Next, we introduce two subgroups of $F(N)$. Let

$$
F^{\prime}:=\left\{f \in F(N) \mid f_{\mid[0, \epsilon]}=i d, f_{\mid[\delta, 1]}=i d, 0<\epsilon, \delta<1\right\}
$$

and the intermediate subgroup

$$
D:=\left\{f \in F(N) \mid f_{\mid[\delta, 1]}=i d, 0<\delta<1\right\} .
$$

These, of course are the same ones considered in [Jol] for $N=2$. It is not hard to see that $F^{\prime}$ and $D$ are normal subgroups of $F(N)$. Actually, when $N=2, F^{\prime}$ is the commutator subgroup (see $\mathrm{Can}$ for $N=2$ and $\mathrm{Br}$ for the general case).

A von Neumann algebra can be thought of as a * subalgebra of bounded operators on some fixed (separable) Hilbert space, that is closed with respect to the weak topology. As shown by John von Neumann the building blocks of the theory are the so-called factors. A von Neumann algebra $M$ inside $B(H)=$ the space of all bounded operators on some Hilbert space $H$ is called a factor if $M \cap M^{\prime}=\mathbb{C}$, where $M^{\prime}$ represents the set of all bounded operators commuting with any element of $M$. There are three types of factors: type I, when $M$ admits minimal projections, e.g., $n \times n$ matrices over $\mathbb{C}$ or the whole $B(H)$; type II, no minimal projection and there exists a unique (semi)finite trace. The factor is called $I I_{1}$ when the trace takes only finite values; type III means not of type I or II. For more on factors and von Neumann algebras we refer the reader to the book [StZ].

Since the beginning of the theory there has been a fruitful interplay between group theory and factors. For example, if $G$ is a countable discrete group with infinite conjugacy classes (i.c.c.), then the left regular representation of $G$ on $l^{2}(G)$ gives rise to a $I I_{1}$ factor, the group von Neumann algebra $\mathcal{L}(G)$, as follows:

Let $l^{2}(G)=\left\{\psi:\left.G \rightarrow \mathbb{C}\left|\sum_{g \in G}\right| \psi(g)\right|^{2}<\infty\right\}$ endowed with the scalar product

$$
\langle\phi, \psi\rangle:=\sum_{g \in G} \phi(g) \overline{\psi(g)} \text {. }
$$


Notice that the Hilbert space $l^{2}(G)$ is generated by the countable collection of vectors $\left\{\delta_{g} \mid g \in G\right\}$. Also, an element $g \in G$ defines a unitary operator $L_{g}$, on $l^{2}(G)$ as follows: $L_{g}(\psi)(h)=\psi\left(g^{-1} h\right)$, for any $\psi \in l^{2}(G)$ and any $h \in G$. (Sometimes, to not burden the notation we will write just $g$ instead of $L_{g}$.) Now, $\mathcal{L}(G)$, the von Neumann algebra generated by $G$ is obtained by taking the wo-closure in $B\left(l^{2}(G)\right)$ of the linear span of the set $\left\{L_{g} \mid g \in G\right\}$. It is a routine exercise to show that $\mathcal{L}(G)$ is a factor provided any element of $G$ has infinite conjugacy classes, and it is of type $I I_{1}$. The map defined by $\operatorname{tr}(L)=\left\langle L\left(\delta_{e}\right), \delta_{e}\right\rangle$, where $e \in G$ is the neutral element and $L \in \mathcal{L}(G)$ is a faithful, normal trace. The canonical trace also determines the Hilbertian norm $|x|_{2}=\operatorname{tr}\left(x^{*} x\right)^{1 / 2}$. It is easy to see that $\operatorname{tr}(g)=0$ for $g \neq e$ and $\operatorname{tr}(e)=1$. Also, for $g \neq h,|g-h|_{2}=2^{1 / 2}$.

The following is an equivalent definition of (inner)amenability:

Definition 2.2. Let $G$ be a (countable discrete) group. If there exists a mean $f$ on the algebra $l^{\infty}(G-\{e\})$, invariant under the action

$$
(g f)(h)=f\left(g^{-1} h\right),
$$

then $G$ is amenable. If the action is taken with respect to conjugation, then $G$ is inner amenable.

Let $M$ be a $I I_{1}$ factor and tr its normal, faithful trace.

Definition 2.3. i) A sequence $\left(x_{n}\right)_{n} \in l^{\infty}(\mathbb{N}, M)$ is a central sequence if

$$
\lim _{n \rightarrow \infty}\left|\left[x, x_{n}\right]\right|_{2}=0
$$

for any $x \in M$, where $[x, y]=x y-y x$.

ii) Two central sequences $\left(x_{n}\right)$ and $\left(y_{n}\right)$ in $M$ are equivalent if

$$
\lim _{n \rightarrow \infty}\left|x_{n}-y_{n}\right|_{2}=0 \text {. }
$$

iii) A central sequence is trivial if it is equivalent to a scalar sequence.

iv) $M$ has property $\Gamma$ of Murray and von Neumann if there exists in $M$ a nontrivial central sequence.

In Efr] it is shown that if the $I I_{1}$ factor $\mathcal{L}(G)$ satisfies property $\Gamma$, then $G$ is inner amenable. A stronger property than $\Gamma$ is the relative McDuff property: a factor $M$ is McDuff if $M$ is isomorphic to $M \otimes R$, where $R$ is the hyperfinite $I I_{1}$ factor ( $R$ can be viewed as $\mathcal{L}(G)$ for an amenable, countable, discrete group $G$ ). D. Bisch extended this property to pairs of $I I_{1}$ factors $1 \in N \subset M$ :

Definition 2.4. The pair $N \subset M$ has the relative McDuff property if there exists an isomorphism $\Phi: M \rightarrow M \otimes R$ such that $\Phi(N)=N \otimes R$.

Finally, we prepare the two results from [Jol] that we are going to use. First, notice that an inclusion $H \subset G$ of groups determines naturally an embedding $1 \in \mathcal{L}(H) \subset \mathcal{L}(G)$ of factors. Also, a semidirect product $H \rtimes_{\alpha} G$ of groups translates into a crossed product $\mathcal{L}(H) \rtimes_{\alpha} G$ in the realm of factors (for more on crossed products see, for example, vDa $)$.

Proposition 2.5 (Proposition 2.4. in [Jol]). Let $G$ a countable i.c.c. group and let $H$ be an i.c.c. subgroup of $G$ with the following property: for every finite subset 
$E$ of $G$ there exist elements $g$ and $h$ in $H-\{e\}$ such that

(1) $x g=g x$ and $x h=h x$ for every $x \in E$;

(2) $g h \neq h g$.

Then the pair $\mathcal{L}(H) \subset \mathcal{L}(G)$ has the relative McDuff property.

Definition 2.6. Let $M$ be a type $I I_{1}$ factor and let $\theta$ be an automorphism of $M$.

i) $\theta$ is centrally trivial if one has for every central sequence $\left(a_{n}\right)_{n}$,

$$
\lim _{n \rightarrow \infty}\left|\theta\left(a_{n}\right)-a_{n}\right|_{2}=0 .
$$

ii) If $G$ is a countable group and if $\alpha$ is an action of $G$ on $M$, then $\alpha$ is called centrally free if $\alpha_{g}$ is not centrally trivial for every $g \in G-\{e\}$.

Proposition 2.7 (Proposition 2.6. in [Jol]). Let $N$ be a McDuff factor of type II with separable predual, let $G$ be an amenable countable group and let $\alpha$ be a centrally free action of $G$ on $N$. Then the pair $N \subset N \rtimes_{\alpha} G$ has the relative McDuff property.

\section{Main Result}

As in [Jol] we will establish that the pairs $\mathcal{L}\left(F^{\prime}\right) \subset \mathcal{L}(D)$ and $\mathcal{L}(D) \subset \mathcal{L}(F(N))$ have the relative McDuff property. In doing so we will first make sure that the group von Neumann algebras involved are $I I_{1}$ factors.

Theorem 3.1. $F^{\prime}$ and $F(N)$ are both i.c.c. groups. Therefore, the von Neumann algebras $\mathcal{L}\left(F^{\prime}\right)$ and $\mathcal{L}(F(N))$ are $I I_{1}$ factors.

Proof. The nice argument that $F^{\prime}$ is i.c.c. is due to Dorin Dutkay: notice first that for any nontrivial $g \in F^{\prime}$ there is a unique $\epsilon$ such that $g_{[0, \epsilon]}=$ id and for any neighborhood $V$ of $\epsilon$ there is $x \in V, x>\epsilon, g(x) \neq x$. We call this unique value $\epsilon_{g}$. Now, we prove that for any $h \in F$,

$$
\epsilon_{h g h^{-1}}=h\left(\epsilon_{g}\right) .
$$

$\epsilon_{h g h^{-1}}$ makes sense because $g \in F^{\prime}$ implies $h g h^{-1} \in F^{\prime}$. Let $x \in\left[0, h\left(\epsilon_{g}\right)\right]$. Then $g h^{-1}(x)=h^{-1}(x)$, hence $h g h^{-1}(x)=x$. The maximality of $\epsilon_{h g h^{-1}}$ implies

$$
\epsilon_{h g h^{-1}} \geq h\left(\epsilon_{g}\right) \text {. }
$$

For the reversed inequality apply the above with the substitutions $h \rightarrow h^{-1}$ and $g \rightarrow$ $h g h^{-1}$. Therefore, (3.1) holds. But, for a fixed nontrivial $g$, the set $\left\{h\left(\epsilon_{g}\right) \mid h \in F^{\prime}\right\}$ is infinite, so that by (3.1) above, the conjugacy class of $g$ is infinite. In conclusion, $F^{\prime}$ is i.c.c.

To prove $F(N)$ is i.c.c we are going to use the elements $A_{d, p}$. Notice first that

$$
A_{d, p}^{-1}(x)=\left\{\begin{array}{lr}
x N^{p}, & 0 \leq x \leq d / N^{p}, \\
x+d-d / N^{p}, & d / N^{p} \leq x \leq 1-d, \\
\left(x+N^{p}-1\right) / N^{p}, & 1-d \leq x \leq 1 .
\end{array}\right.
$$

We assume $f \in F(N)$ is nontrivial and that in a neighborhood of $x=0$ its slope is positive. Once we prove that its conjugacy class is infinite the class of its inverse follows infinite, so there is no loss of generality in assuming a positive slope around the origin. For any $p \in \mathbb{Z}, p \neq 0$ we will find a large $\alpha$ such that for any $k>l>\alpha$,

$$
A_{d, p}^{-1} f A_{d, p} \neq A_{\tilde{d}, p}^{-1} f A_{\tilde{d}, p},
$$

where $d=1 / N^{k}$ and $\tilde{d}=1 / N^{l}$. This relation clearly shows that the conjugacy class of $f$ is infinite. 
From the definition of $F(N)$ there is an $N$-adic $d_{1}$ such that $f_{\mid\left[0, d_{1}\right]}(x)=N^{n} x$, where, by our assumption $n>0$ (the case $n=0$ is taken care of by the previous argument, i.e., when $f$ is trivial around $x=0$ ). Now choose $\alpha$ large enough such that the following inequalities hold:

$$
\frac{1}{N^{\alpha+p}}<d_{1}, \frac{N^{n}}{N^{\alpha+p}}<1-\frac{1}{N^{\alpha}} .
$$

For $k>l>\alpha$ consider the $N$-adic numbers $d$ and $\tilde{d}$. If (3.2) were not true, then evaluating at $x=d=1 / N^{k}$ we obtain

$$
A_{d, p}^{-1} f\left(\frac{1}{N^{k+p}}\right)=A_{\tilde{d}, p}^{-1} f\left(\frac{1}{N^{k+p}}\right) .
$$

Because $1 / N^{k+p} \in\left[0, d_{1}\right]$, the equality becomes

$$
A_{d, p}^{-1}\left(\frac{N^{n}}{N^{k+p}}\right)=A_{\tilde{d}, p}^{-1}\left(\frac{N^{n}}{N^{k+p}}\right) .
$$

Because of the choices of $n, \alpha, k$ and $l$, we have

$$
\frac{1}{N^{i+p}}<\frac{N^{n}}{N^{i+p}}<\frac{N^{n}}{N^{\alpha+p}}<1-\frac{1}{N^{\alpha}}<1-\frac{1}{N^{i}}
$$

where $i \in\{k, l\}$. This shows that

$$
x:=\frac{N^{n}}{N^{k+p}} \in\left[\frac{d}{N^{p}}, 1-d\right] .
$$

Using formula of $A_{d, p}^{-1}$, equation (3.3) can be rewritten as

$$
x+d-\frac{d}{N^{p}}=A_{\tilde{d}, p}^{-1}(x) .
$$

Because of the way $\alpha$ has been chosen, we get $x<1-\tilde{d}$, so that there are only two cases to discuss:

$x \in\left[\frac{\tilde{d}}{N^{p}}, 1-\tilde{d}\right]$. Relation (3.4) easily implies $d=\tilde{d}$, which of course is not allowed.

$x \in\left[0, \frac{\tilde{d}}{N^{p}}\right]$. Using the formula for $A_{\tilde{d}, p}^{-1}$ on $\left[0, \frac{\tilde{d}}{N^{p}}\right]$ and putting $x=\frac{N^{n}}{N^{k+p}}$ and $d=\frac{1}{N^{k}}$ back in (3.4) we obtain

$$
\frac{N^{n}}{N^{k+p}}+\frac{1}{N^{k}}-\frac{1}{N^{k+p}}=\frac{N^{n}}{N^{k}}
$$

which reduces (using $n \neq 0$ ) to $N^{k+p}=N^{k}$. This would imply $p=0$, a value that we avoid.

In conclusion, (3.2) is true and we finish the proof.

We prove the following lemma, useful for scaling down graphs of elements in $F(N)$ and still remaining in $F(N)$ :

Lemma 3.2. Let $0<\delta<\epsilon<1$ be $N$-adic numbers such that $\epsilon-\delta \in N^{\mathbb{Z}}$. Then there exists $f \in F(N)$ with $f_{\mid[0, \delta]}(x)=x$ and $f_{\mid[\epsilon, 1]}(x)=x$ and $f$ has no fixed points in $(\delta, \epsilon)$.

Proof. Take $r:[\delta, \epsilon] \rightarrow[0,1]$ defined by $r(x)=\frac{x}{\epsilon-\delta}-\frac{\delta}{\epsilon-\delta}$. For $d$, a nonzero $N$-adic and $p \neq 0$, consider the following homeomorphism:

$$
f(x)= \begin{cases}x, & 0 \leq x \leq \delta \\ r^{-1} A_{d, p} r(x), & \delta \leq x \leq \epsilon, \\ x, & \epsilon \leq x \leq 1\end{cases}
$$


When the derivative exists, $f^{\prime}(x)=A_{d, p}^{\prime}(r(x))$, for $x \in(\delta, \epsilon)$. Thus we obtain $f \in F(N)$. Also, there can be no fixed point of $f$ in $(\delta, \epsilon)$ as $A_{d, p}$ has no other fixed points besides 0 and 1 .

Next, we are going to check the hypotheses of Proposition 2.4 from [Jol]:

Proposition 3.3. For every finite subset $\left\{g_{1}, g_{2}, \ldots, g_{n}\right\}$ of $D$ there exist nontrivial distinct elements $g$ and $h$ of $F^{\prime}$ such that

(1) $g_{i} g=g g_{i}$ and $g_{i} h=h g_{i}$ for all $i \in\{1,2, \ldots, n\}$,

(2) $h g \neq g h$.

Proof. Because $g_{i} \in D$, there exists $\delta$ such that $g_{i \mid[\delta, 1]}=$ id for all $i \in\{1,2, \ldots, n\}$. Also, we may take $\delta N$-adic. For $\epsilon_{1}$ chosen such that $\epsilon_{1}-\delta \in N^{\mathbb{Z}}$ apply the lemma above: there exists $g \in F^{\prime}$ with $g_{[0, \delta]}=$ id, having no fixed points inside $\left(\delta, \epsilon_{1}\right)$. An easy check shows $g_{i} g=g g_{i}$. Now, for $\epsilon_{2}>\epsilon_{1}$ such that $\epsilon_{2}-\delta \in N^{\mathbb{Z}}$ we find, using the lemma again, an $h \in F^{\prime}$ with the very same properties. Therefore, (1) is satisfied. Now, for any $f \in F^{\prime}$ let $\epsilon_{h}$ be the smallest $\epsilon$ such that $f_{\mid[\epsilon, 1]}=$ id and for any neighborhood $V$ of $\epsilon$ there exists a $x \in V, x<\epsilon$ with $f(x) \neq x$. For $g$ and $f$ found above we clearly have $\epsilon_{1}=\epsilon_{g}$ and $\epsilon_{2}=\epsilon_{h}$. Moreover, as in the proof of Theorem 3.1] the following equality holds true: $\epsilon_{h g h^{-1}}=h\left(\epsilon_{g}\right)$. If (2) were not satisfied, then we would obtain $\epsilon_{1}=h\left(\epsilon_{1}\right)$, which contradicts the fact that $h$ has no fixed point inside $\left(\delta, \epsilon_{2}\right)$.

Corollary 3.4. The pair $\mathcal{L}\left(F^{\prime}\right) \subset \mathcal{L}(D)$ has the relative McDuff property.

Proof. Apply Proposition 2.4. of [Jol].

Remark 3.5. We will continue with the ideas in Jol to show that the pair $\mathcal{L}(D) \subset$ $\mathcal{L}(F(N))$ has the relative McDuff property. Notice first that the general Thompson group can be realized as a semiproduct $D \rtimes_{\alpha} \mathbb{Z}$ where the action $\alpha$ is defined as follows: choose $x_{0} \in F(N)$ such that its first piece of graph is trivial and the slope of the last piece is $N$ (it is elementary to construct such an element in $F(N)$; see the proof of Proposition 3.8 below). Then the action $\alpha(n)(f)=x_{0}^{n} f x_{0}^{-n}$ is well defined on $D$. Also, any element of $F(N)$ can be written as $f x_{0}^{-n}$ for some $f \in D$ and $n \in \mathbb{Z}$, and therefore the map $(f, n) \in D \rtimes_{\alpha} \mathbb{Z} \rightarrow f x_{0}^{-n}$ is a group isomorphism. For a central sequence in $\mathcal{L}(D)$ we choose the unitary operators corresponding to the following sequence $\left(a_{n}\right)_{n} \subset D$ : let $\left(d_{n}\right)_{n}$ and $\left(\overline{d_{n}}\right)_{n}$ be two sequences of $N$-adic numbers in $[0,1]$ such that $d_{n}<\overline{d_{n}}$ are consecutives with $d_{n} \rightarrow 1$. Applying the scaling-down lemma we obtain a nontrivial $a_{n} \in F^{\prime}$. Moreover, for any $g$ in a finite subset of $D$ and for large $n, a_{n}$ commutes with $g$ (see the proof of Proposition 3.3).

Notice that $F^{\prime}$ is inner amenable; in particular, by the Corollary above, $\mathcal{L}\left(F^{\prime}\right)$ has property $\Gamma$, so that me may apply the result in Efr. to conclude $F^{\prime}$ is inner amenable.

Theorem 3.6. The pair $\mathcal{L}(D) \subset \mathcal{L}(F(N))$ has the relative McDuff property.

Proof. We will make use of Proposition 2.6 in [Jol]: it suffices to check that the action $\alpha$ is centrally free. Having already a central sequence given by $\left(a_{n}\right)_{n}$, it is enough to show $\lim _{n}\left|\alpha^{m}\left(a_{n}\right)-a_{n}\right|_{2}>0$, for all $m \neq 0$. Notice that for $g \neq h$ in a i.c.c group, we have $|g-h|_{2}=2^{1 / 2}$, hence it suffices to prove that $\alpha^{m}\left(a_{n}\right)$ is not 
equal to $a_{n}$, for sufficiently large $n$. Using the notation in the proof of Theorem 3.1 (see relation (3.1)) we get $\epsilon_{a_{n}}=d_{n}$. If $\alpha^{m}\left(a_{n}\right)=a_{n}$, then we obtain

$$
\epsilon_{x_{0}^{m} a_{n} x_{0}^{-m}}=\epsilon_{a_{n}} .
$$

Applying (3.1),

$$
x_{0}^{m}\left(d_{n}\right)=d_{n} .
$$

The last equality cannot happen though, as $x_{0}^{m}$ has slope equal to $N^{m}$ near $x=1$ and $d_{n} \rightarrow 1$.

Corollary 3.7. For any integer $N \geq 2$, the generalized Thompson group $F(N)$ is inner amenable.

Proof. From the theorems above $\mathcal{L}(F(N))$ has property $\Gamma$.

The next proposition establishes an exact sequence that allows us once again to conclude $F(N)$ is inner amenable. For $N=2$ this proposition specializes in Theorem 4.1. of Can (except the part about the commutator subgroup; see also Che ) and also appears in a more general form in $\mathrm{St}$.

Proposition 3.8. One has the short exact sequence

$$
1 \rightarrow F^{\prime} \rightarrow F(N) \rightarrow \mathbb{Z}^{2} \rightarrow 1 .
$$

Proof. We prove that the following group morphism is onto: $\phi: F(N) \rightarrow \mathbb{Z}^{2}$, $\phi(f)=(a, b)$, where $f$ has slope $N^{a}$ near $x=0$ and slope $N^{b}$ near $x=1$. It suffices to show that there exist $f_{1}$ and $f_{2}$ in $F(N)$ such that $\phi\left(f_{1}\right)=(1,0)$ and $\phi\left(f_{2}\right)=(0,1)$. Let $p>0$ and $d:=1 / N^{p}$ such that $d(N+1)<1$. Define now

$$
f_{1}(x)=\left\{\begin{array}{lr}
N x, & 0 \leq x \leq d, \\
\frac{x}{N}+N d-\frac{d}{N}, & d \leq x \leq d(N+1), \\
x, & d(N+1) \leq x \leq 1 .
\end{array}\right.
$$

Clearly, $f_{1} \in F(N)$ and $\phi\left(f_{1}\right)=(0,1) ; f_{2}$ can be obtained by applying a symmetry to $f_{1}$. In conclusion $\phi$ is onto. Notice that its kernel is exactly the normal subgroup $F^{\prime}$.

Question: Following $[\mathrm{Br}$ in a particular case we obtain that $F(2)=F$ is not isomorphic to $F(3)$. Is it true that $\mathcal{L}(F(2)) \cong \mathcal{L}(F(3))$ ? Notice that if these factors are not isomorphic, then by the uniqueness of the hyperfinite $I I_{1}$ factor at least one of the groups $F$ or $F(3)$ follows nonamenable.

\section{ACKNOWLEDGEMENTS}

We would like to thank Dorin Dutkay for many useful suggestions. We also thank Professor Sean Cleary for indicating the right references on the generalized Thompson group. We express our gratitude to Professor Florin Radulescu for his constant support. 


\section{REFERENCES}

[Br] K.S. Brown, Finiteness Properties of Groups, J. Pure Appl. Algebra, 44 (1986), 45-75. MR 0885095 (88m:20110)

[Can] J.W. Cannon, W.J. Floyd, and W.R. Parry, Introductory Notes on Richard Thompson's Groups, L'Enseignement Mathematique, 42 (1996), pp. 215-256. MR1426438 (98g:20058)

[Che] T.G. Ceccherini-Silberstein, Around Amenability, Journal of Mathematical Sciences, Vol. 106, No. 4, 2001. MR1871137 (2003d:43003)

[vDa] A. van Daele, Crossed Products of von Neumann Algebras, Lecture Notes Series, London Math. Soc., 1978.

[DutP] D. Dutkay, G. Picioroaga, The von Neumann Algebra of the Canonical Equivalence Relation of the Thompson Group , 2004, arxiv id math.OA/0403332.

[Efr] E.G. Effros, Property $\Gamma$ and Inner Amenability, Proc. Amer. Math. Soc., 47 (1975), 483486. MR0355626 (50:8100)

[Hig] G. Higman, Finitely Presented Infinite Simple Groups, Notes on Pure Math., vol. 8, Australian National University, Canberra, 1974. MR0376874 (51:13049)

[Jol1] P. Jolissaint, Moyennabilite Interieure du Groupe F de Thompson, C.R. Acad. Sci. Paris, Serie I, 325 (1997), 61-64. MR1461398 (98j:20049)

[Jol] P. Jolissaint, Central Sequences in the Factor Associated with the Thompson's Group $F$, Annales de l'institut Fourier, tome 48, no. 4 (1998), pp. 1093-1106. MR1656009 (2000b:46108)

[St] M. Stein, Groups of Piecewise Linear Homeomorphisms, Trans. Amer. Math. Soc., 332 (1992), no. 2, 477-514. MR1094555 (92k:20075)

[StZ] S. Stratila, L. Zsido, Lectures on von Neumann Algebras, Bucuresti, Romania, Editura Academiei; Tunbridge Well, Kent, England, Abacus Press, 1979. MR0526399 (81j:46089)

Department of Mathematics, The University of Iowa, 14 Maclean Hall, Iowa City, IowA 52242-1419

E-mail address: gpicioro@math.uiowa.edu

Current address: Department of Mathematics and Computer Science, University of Southern Denmark, Campusvej 55, DK-5230, Odense M, Denmark

E-mail address: gpicioro@imada.sdu.dk 